Keywords: cancer-specific antigens; cancer testes antigens; HAGE; early breast cancer; translation therapeutics; prognostic and predictive factor; immunotherapy vaccine

\title{
HAGE (DDX43) is a biomarker for poor prognosis and a predictor of chemotherapy response in breast cancer
}

\author{
T M A Abdel-Fatah ${ }^{1}$, S E B McArdle ${ }^{2}$, C Johnson $^{2}$, P M Moseley ${ }^{1}$, G R Ball ${ }^{2}$, A G Pockley ${ }^{2}$, I O Ellis ${ }^{3}$, \\ R C Rees ${ }^{2}$ and S Y T Chan*,1 \\ ${ }^{1}$ Department of Clinical Oncology, University of Nottingham City Hospital NHS Trust, Nottingham, UK; ${ }^{2}$ John van Geest Cancer \\ Research Centre, Nottingham Trent University, Nottingham, UK and ${ }^{3}$ Department of Histopathology, University of Nottingham \\ City Hospital NHS Trust, Nottingham, UK
}

Background: HAGE protein is a known immunogenic cancer-specific antigen.

\begin{abstract}
Methods: The biological, prognostic and predictive values of HAGE expression was studied using immunohistochemistry in three cohorts of patients with $B C(n=2147)$ : early primary (EP-BC; $n=1676)$; primary oestrogen receptor-negative (PER-BC; $n=275)$ treated with adjuvant anthracycline-combination therapies (Adjuvant-ACT); and primary locally advanced disease (PLA-BC) who received neo-adjuvant anthracycline-combination therapies (Neo-adjuvant-ACT; $n=196$ ). The relationship between HAGE expression and the tumour-infiltrating lymphocytes (TILs) in matched prechemotherapy and postchemotherapy samples were investigated.
\end{abstract}

Results: Eight percent of patients with EP-BC exhibited high HAGE expression (HAGE + ) and was associated with aggressive clinico-pathological features (Ps $<0.01$ ). Furthermore, HAGE + expression was associated with poor prognosis in both univariate and multivariate analysis (Ps $<0.001$ ). Patients with HAGE + did not benefit from hormonal therapy in high-risk ER-positive disease. HAGE + and TILs were found to be independent predictors for pathological complete response to neoadjuvant-ACT; $P<0.001$. A statistically significant loss of HAGE expression following neoadjuvant-ACT was found $(P=0.000001)$, and progression-free survival was worse in those patients who had HAGE + residual disease $(P=0.0003)$.

Conclusions: This is the first report to show HAGE to be a potential prognostic marker and a predictor of response to ACT in patients with BC.

Breast cancer (BC) is the most common and leading cause of cancer deaths in women. Despite the improved outcomes which have been achieved by earlier detection and recent advances in therapy, recurrence still occurs in over $20 \%$ of patients (Early Breast Cancer Trialists' Collaborative Group et al, 2008; Dotan and Goldstein, 2010; Gluck and Mamounas, 2010). It is therefore becoming increasingly important to identify biomarkers that can aid in assessing a patient's risk and response to therapy and which might also act as novel targets for immunotherapies (Harris et al, 2007; Harbeck et al, 2010). Although identifying tumour-associated antigens that induce adaptive Tcell immunity is a prerequisite for establishing effective immunotherapy strategies, there is currently a lack of suitable candidates for the development of such treatments in BC (Mathieu et al, 2007).

The helicase antigen known as HAGE was first identified in a sarcoma cell line using representational difference analysis (Martelange et al, 2000). HAGE was subsequently shown to be expressed in many haematological and solid tumour samples but not in any of the normal tissues tested, except testis (Martelange et al, 2000; Adams et al, 2002; Mathieu et al, 2010) - it was 
therefore classified as a Cancer Testes Antigen (CTA). We have previously reported that HAGE protein is required for the proliferation of cancer cells and that it is immunogenic (Adams et al, 2002; Mathieu et al, 2007).

To date, the clinico-pathological and prognostic significance of HAGE expression in BC remains unknown. We have therefore assessed the clinico-pathological and prognostic and predictive significance of HAGE expression in BC using three wellcharacterized cohorts of patients $(n=2147)$.

\section{PATIENTS AND METHODS}

Study populations. Consent was obtained from all the patients as per hospital's Standard of Care. This included a clause which permitted the use of excess tissue in research projects that had been approved by both the Local Research Committee and the Hospital Research and Innovations Department.

All work was conducted under the study 'Development of a molecular genetic classification of breast cancer' and approved by the Nottingham Research Ethics Committee (Ref: C1080301) and the Hospital Research and Innovations Department (Ref: 03HI01).

Early primary BC (EP-BC) cohort. The study was performed in a 1676 consecutive series of patients with primary invasive BC who were diagnosed between 1986 and 1999 (Elston and Ellis, 1991; Pinder et al, 1994). Patients received standard surgery (mastectomy or wide local excision) with radiotherapy. Before 1989, patients did not receive either endocrine or chemotherapy. After 1989, adjuvant-therapy was scheduled on the basis of the Nottingham Prognostic Index (NPI), oestrogen receptor- $\alpha$ (ER- $\alpha$ ) and menopausal status. Patients with NPI scores $<3.4$ (low risk) did not receive adjuvant-therapy. Premenopausal patients with NPI scores $\geqslant 3.4$ (high risk) received cyclophosphamide, Methotrexate and 5-Flourouracil (CMF) combination chemotherapy, and patients with ER- $\alpha$-positive tumours were also offered Tamoxifen for 5 years. The minimum follow-up period was 123 months. Data were randomised into two equal cohorts using a double random number sort. Alternate cases were put into a training set $(n=838)$ and a test set $(n=838)$. Both cohorts were balanced with regards to HAGE expression, primary clinicopathological features, treatment and survival outcome (Supplementary Table S1).

Primary ER-negative BC (PER-BC). In order to evaluate the value of HAGE protein expression as a biomarker in the context of current combination cytotoxic chemotherapy, we also analysed its expression in an independent series of 275 patients with PER-BC who had been diagnosed and managed at the same institution between 1999 and 2007, 141 of whom were treated with adjuvantACT. Comprehensive follow-up data were available for 275 patients (median $=89$ months, mean $=86$ months; Supplementary Table S2).

Primary locally advanced BC (PLA-BC). Tumour-infiltrating lymphocytes (TILs) and HAGE expression were evaluated in pairmatched prechemotherapy core biopsies and postchemotherapy surgical specimens from 196 female patients with PLA-BC (stage IIIA-C) treated with neoadjuvant-ACT, and their relationship(s) with response to treatment and survival were investigated. These patients were diagnosed by needle core biopsy at the Nottingham University City Hospital between 1996 and 2010. A pathological complete response ( $\mathrm{pCR}$ ) was defined as the absence of any residual invasive carcinoma at both the primary site and in axillary lymph nodes (LNs). On average, 16 breast-blocks and all submitted LNs were examined for each case before a diagnosis of pCR was reached. Detailed patient demographics and clinico-pathological characteristics were routinely assessed and regularly updated (Supplementary Table S3).
Pathology assessment of TILs. Histopathological analysis of the TILs was performed on haematoxylin and eosin-stained sections adopting the protocol by Denkert et al (2010). In summary, intratumoral lymphocytes (iTu-Ly) were defined as intraepithelial mononuclear cells within tumour cell nests or in direct contact with tumour cells and were reported as the percentage of the tumour epithelial nests that contain infiltrating lymphocytes. Stromal lymphocytes (str-Ly) were defined as the percentage of tumour stroma area that contains a lymphocytic infiltrate without direct contact to tumour cells. Histopathological analysis was performed by two pathologists (TAF and IOE) who were blinded to clinical and response data; the mean was used for analysis. TILs was classified into three categories: (1) TILs predominant infiltrate defined as the presence of either intratumoral lymphocytes in $>60 \%$ of tumour cell nests or lymphocytes in $>60 \%$ of the stromal area, (2) focal TILs infiltrate included detectable mononuclear cells $>10 \%$ but $<60 \%$ of str-Ly or iTu-Ly and (3) no or minimal TILs defined as no detectable or presence of $\leqslant 10 \%$ mononuclear cells in tumour cell nests and tumour stroma.

Survival data. Survival data, including survival time, disease-free survival (DFS) and development of loco-regional and distant metastases (DM), were maintained on a prospective basis. DFS was defined as the number of months from diagnosis to the occurrence of recurrence or DM relapse. BC-specific survival (BCSS) was defined as the number of months from diagnosis to the occurrence of BC-related death. Survival was censored if the patient was still alive, lost to follow-up or died from other causes. The Reporting Recommendations for Tumor Marker Prognostic Studies (REMARK) criteria (McShane et al, 2005) were followed throughout this study.

Tissue microarrays (TMAs) and immunohistochemistry (IHC). Tumours from both EP-BC and PER-BC cohorts, as well as postsurgical specimens from patients with PLA-BC, were incorporated into TMAs. These were constructed using six replicate $0.6-\mathrm{mm}$ cores from the centre and periphery of the tumours of each patient. In addition, full-face sections from the diagnostic prechemotherapy core biopsy were used for those PLA-BC cases that were treated with neoadjuvant-ACT. The TMAs and full-face sections were IHC profiled using antibodies for HAGE and other antibodies (Supplementary Table S4), as previously described (Abdel-Fatah et al, 2010a,b). IHC staining was performed using a Novolink Detection kit according to the manufacturer's protocol (Leica Microsystems, Wetzlar, Germany). For this, sections were incubated overnight at $4{ }^{\circ} \mathrm{C}$ with $1 / 175$ dilution of custom-made rabbit anti-HAGE mono-specific polyclonal antibody (Mathieu et al, 2010). The IHC was validated using a commercially available antibody that was developed and validated as part of the Human Protein Atlas (HPA) project (anti-DDX43; HPA031381, SigmaAldrich, St Louis, MO, USA); in this case, sections were incubated overnight at $4{ }^{\circ} \mathrm{C}$ at $1 / 100$ dilution (Supplementary Figure S1).

Expression of HER2, ER and PR was assessed according to the American Society of Clinical Oncology/College of American Pathologists (ASCO/CAP) guidelines (Wolff et al, 2007; Hammond et al, 2010).

To validate the use of TMAs for immuno-phenotyping, full-face sections of 40 cases were stained, and the protein expression levels were compared. The concordance between TMAs and full-face sections was excellent using Cohen's $\kappa$ statistical test for categorical variables $(\kappa=0.8)$. Positive and negative (omission of the primary antibody and IgG-matched serum) controls were included in each run.

Evaluation of HAGE immunohistochemical staining. Tumour cores were evaluated by two pathologists (co-authors: TAF, IOE) who were blinded to the clinico-pathological characteristics of patients in two different settings. Whole-field inspection of the 
core was scored, and intensities of both nuclear and cytoplasmic staining were grouped as follows: $0=$ no staining, $1=$ weak staining, $2=$ moderate staining, and $3=$ strong staining. The percentage of each category was estimated and due to intratumoral and intertumoral heterogeneity of the staining, the average percentage among six cores was calculated and high HAGE $(\mathrm{HAGE}+)$ expression was defined as the presence of strong cytoplasmic and/or nuclear staining in $>10 \%$ of malignant cells (Figure 1A). Intraobserver ( $\kappa>0.8$; Cohen's $\kappa$ test) and interobserver $(\kappa>0.8$; using multi-rater $\kappa$ tests) observer agreements were excellent. In cases where discordant results were obtained, the slides were re-evaluated by both pathologists together, and a consensus was reached.

Statistical analysis. Data analysis was performed by (TAF, GRB and SYC) who were blinded to clinical and response data using SPSS (Version 17, Chicago, IL, USA). Where appropriate, Pearson's Chi-square and Student's tests were used. Positivity for HAGE protein both before and after chemotherapy was calculated and compared using McNemar's test. Significance was defined at $P<0.05$.

Cumulative survival probabilities were estimated using the Kaplan-Meier method, and the differences between survival rates were tested for significance using the log-rank test. Multivariate analysis for survival was performed using the Cox proportional hazard model. The proportional hazards assumption was tested using standard log-log plots. Hazard ratios (HRs) and 95\% confidence intervals (95\% CIs) were estimated for each variable. All tests were two-sided with a $95 \% \mathrm{CI}$, and a $P$-value $<0.05$ was considered to be indicative of statistical significance. A stringent $P$-value $<0.01$ was considered to indicate statistical significance for multiple comparisons.

\section{RESULTS}

\section{EP-BC patient group - training set $(n=838)$}

Clinico-pathological significance of HAGE expression. Eight percent (69 out of 838) of tumours examined were found to be positive for HAGE expression (HAGE + ; Figure 1A). In invasive BC, HAGE + expression was significantly associated with aggressive and adverse pathological features (Table 1), including high grade, absence of hormonal receptors (ER $-/ \mathrm{PR}-$ ), absence of the anti-apoptotic $\mathrm{Bcl} 2(\mathrm{Bcl} 2-)$, presence of the triple-negative phenotype (TNBC) and overexpression of HER2 (HER2 + ). Interestingly, none of the invasive lobular carcinomas were $\mathrm{HAGE}+(0$ out of 69$)$.

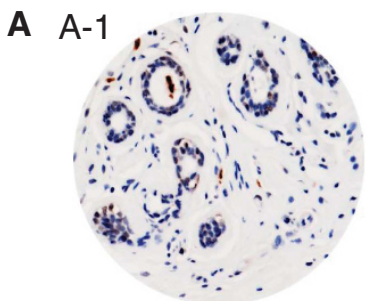

Normal breast tissue

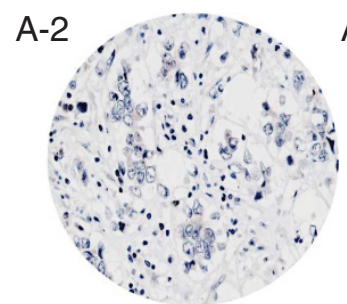

Negative HAGE expression

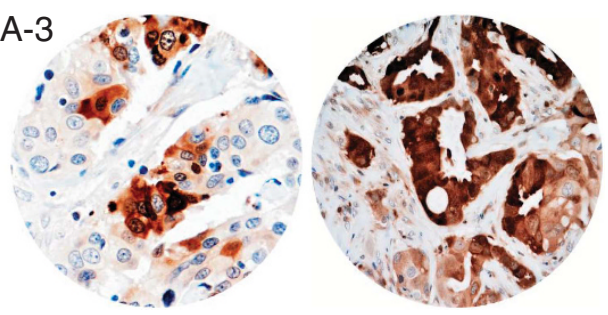

High HAGE expression

B

Clinical outcome of the training set
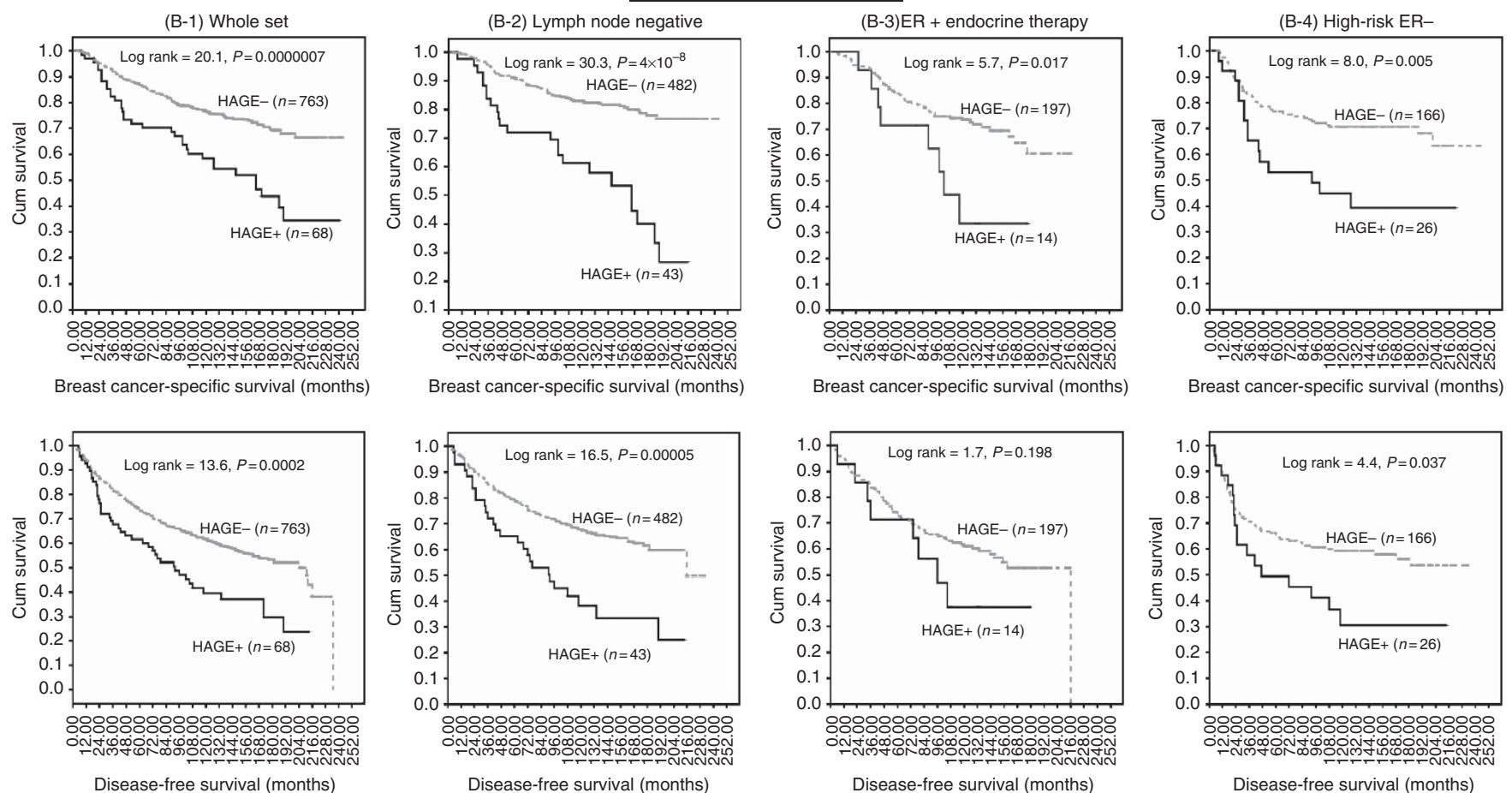

Figure 1. The clinical outcome of HAGE protein expression in the training set of primary early breast cancer. (A) (1-3) Photomicrographs showing weak nuclear expression of HAGE in normal breast tissue (A-1), breast cancer tissue (A2-3) showing negative expression of HAGE in neoplastic cells (A-2), positive expression of HAGE in neoplastic cells (A-3) (magnification $\times 200$ ). (B) (1-4) Kaplan-Meier curves showing the relationship between HAGE expression and breast cancer-specific and disease-free survival in the training set of primary early breast cancer (PE-BC). See text for details. 
Table 1. Clinico-pathological characteristics of HAGE protein expression in the discovery and test sets

Discovery set $(n=838), n(\%)$

Test set $(n=838)$

Variables

HAGE-negative HAGE-positive

$(n=769)$

$(n=69)$

\section{Adjusted \\ $P$-value}

Pathological parameters

Tumour size

$\mathrm{T} 1 \mathrm{a}+\mathrm{b}(\leqslant 1.0)$

T1 c (>1.0-2.0)

T2 (>2.0-5.0)

T3 $(>5)$

Lymph node stage

\section{Negative}

Positive (1-3 nodes)

Positive ( $>3$ nodes)

Tumour type

IDC-NST

Medullary/atypical

Tubular carcinoma

Invasive lobular carcinoma

Others

Tumour grade ${ }^{a}$

\begin{tabular}{l|l} 
G1 & $138(18.1)$
\end{tabular}

G2

G3

$268(35.2)$
87 (11.4)

395 (51.8)

255 (33.5)

25 (3.3)

484 (63.4)

$211(27.7)$

68 (8.9)

$385(58.6)$

12 (1.8)

138 (21.0)

81 (12.3)

41 (6.2)
0.1

$44(63.8)$
$21(30.4)$
$4(5.8)$

42 (66.7)

1 (1.6)

14 (22.2)

$0(0)$

$6(9.5)$
3 (4.3)

$38(55.1)$

$28(40.6)$

$0(0)$

\begin{tabular}{|r|r}
$90(11.7)$ \\
$378(49.3)$ \\
$283(36.9)$ \\
$15(2.0)$
\end{tabular}

0.642

$474(61.7)$
$228(29.7)$
$66(8.6)$

0.052

11 (15.9)

11 (15.9)

47 (68.1)

\section{7 (55.4)}

19 (2.9)

$133(20.1)$

$73(11.0)$

$70(10.6)$
5 (7.2)

37 (53.6)

$26(37.7)$

1 (1.4)
0.693

Adjusted

$P$-value

\section{Hormonal receptors}

Oestrogen receptor

\begin{tabular}{|c|c|c|c|c|c|c|}
\hline $\begin{array}{l}\text { Negative } \\
\text { Positive }\end{array}$ & $\begin{array}{l}186(25.1) \\
556(74.9)\end{array}$ & $\begin{array}{l}28(40.6) \\
41(59.4)\end{array}$ & $0.005^{b}$ & $\begin{array}{l}176(23.2) \\
581(76.8)\end{array}$ & $\begin{array}{l}31(46.3) \\
36(53.7)\end{array}$ & $0.00003^{b}$ \\
\hline \multicolumn{7}{|c|}{ Progesterone receptor } \\
\hline $\begin{array}{l}\text { Negative } \\
\text { Positive }\end{array}$ & $\begin{array}{l}295(41.5) \\
416(58.5)\end{array}$ & $\begin{array}{l}39(56.5) \\
30(43.5)\end{array}$ & $0.016^{b}$ & $\begin{array}{l}273(38.1) \\
444(61.9)\end{array}$ & $\begin{array}{l}40(58.8) \\
28(41.2)\end{array}$ & $0.001^{b}$ \\
\hline \multicolumn{7}{|c|}{ Androgen receptor } \\
\hline $\begin{array}{l}\text { Negative } \\
\text { Positive }\end{array}$ & $\begin{array}{l}210(35.2) \\
386(64.8)\end{array}$ & $\begin{array}{l}24(42.1) \\
33(57.9)\end{array}$ & 0.301 & $\begin{array}{l}221(37.0) \\
376(63.0)\end{array}$ & $\begin{array}{l}23(36.5) \\
40(63.5)\end{array}$ & 0.936 \\
\hline
\end{tabular}

Proliferation/cell cycle regulators

Mitotic index

\begin{tabular}{l|l|l|l|r|r|} 
M1 (low; mitoses <10) & $298(39.4)$ & $14(20.3)$ & $0.002^{\mathbf{b}}$ & $288(38.0)$ & $10(14.5)$ \\
M2 (medium; mitoses 10-18) & $140(18.5)$ & $12(17.4)$ & & $142(18.7)$ \\
M3 (high; mitoses>18) & $318(42.1)$ & $43(62.3)$ & & $328(43.3)$ \\
\hline
\end{tabular}

Ki67

\begin{tabular}{|c|c|c|c|c|c|c|}
\hline $\begin{array}{l}\text { Negative } \\
\text { Positive }\end{array}$ & $\begin{array}{l}241(38.7) \\
382(61.3)\end{array}$ & $\begin{array}{l}16(23.2) \\
53(76.8)\end{array}$ & $0.011^{b}$ & $\begin{array}{l}249(38.7) \\
395(61.3)\end{array}$ & $\begin{array}{l}17(25.0) \\
51(75.0)\end{array}$ & $0.027^{b}$ \\
\hline \multicolumn{7}{|l|}{ KIF2C } \\
\hline $\begin{array}{l}\text { Negative } \\
\text { Positive }\end{array}$ & $\begin{array}{l}218(42.7) \\
292(57.3)\end{array}$ & $\begin{array}{r}9(20.0) \\
36(80.0)\end{array}$ & $0.003^{b}$ & $\begin{array}{l}203(38.7) \\
322(61.3)\end{array}$ & $\begin{array}{r}9(17.0) \\
44(83.0)\end{array}$ & $0.002^{b}$ \\
\hline \multicolumn{7}{|l|}{ SPAG5 } \\
\hline $\begin{array}{l}\text { Negative } \\
\text { Positive }\end{array}$ & $\begin{array}{l}482(80.7) \\
115(19.3)\end{array}$ & $\begin{array}{l}38(66.7) \\
19(33.3)\end{array}$ & $0.012^{b}$ & $\begin{array}{l}494(82.9) \\
102(17.1)\end{array}$ & $\begin{array}{l}37(66.1) \\
19(33.9)\end{array}$ & $0.004^{b}$ \\
\hline \multicolumn{7}{|l|}{ TOP2A } \\
\hline $\begin{array}{l}\text { Negative } \\
\text { Positive }\end{array}$ & $\begin{array}{l}251(46.2) \\
292(53.8)\end{array}$ & $\begin{array}{l}23(46.0) \\
27(54.0)\end{array}$ & 0.976 & $\begin{array}{l}254(47.6) \\
280(52.4)\end{array}$ & $\begin{array}{l}19(33.9) \\
37(66.1)\end{array}$ & 0.066 \\
\hline \multicolumn{7}{|l|}{ P16 } \\
\hline $\begin{array}{l}\text { Negative } \\
\text { Positive }\end{array}$ & $\begin{array}{r}460(87.1) \\
68(12.9)\end{array}$ & $\begin{array}{r}41(82.0) \\
9(18.0)\end{array}$ & 0.308 & $\begin{array}{r}455(87.7) \\
64(12.3)\end{array}$ & $\begin{array}{l}40(71.4) \\
16(28.6)\end{array}$ & $0.002^{b}$ \\
\hline
\end{tabular}


Table 1. (Continued)

Discovery set $(n=838), n(\%)$

Test set $(n=838)$

\begin{tabular}{|l|c|c|c|c|c|c|}
\hline Variables & $\begin{array}{c}\text { HAGE-negative } \\
(n=769)\end{array}$ & $\begin{array}{c}\text { HAGE-positive } \\
(n=69)\end{array}$ & $\begin{array}{c}\text { Adjusted } \\
P \text {-value }\end{array}$ & $\begin{array}{c}\text { HAGE-negative } \\
(n=769)\end{array}$ & $\begin{array}{c}\text { HAGE-positive } \\
(n=69)\end{array}$ & $\begin{array}{c}\text { Adjusted } \\
P \text {-value }\end{array}$ \\
\hline
\end{tabular}

P53/apoptosis pathways

P53

\begin{tabular}{|c|c|c|c|c|c|c|}
\hline $\begin{array}{l}\text { Negative } \\
\text { Positive }\end{array}$ & $\begin{array}{l}495(80.9) \\
117(19.1)\end{array}$ & $\begin{array}{l}40(70.2) \\
17(29.8)\end{array}$ & 0.053 & $\begin{array}{l}484(79.5) \\
125(20.5)\end{array}$ & $\begin{array}{l}41(73.2) \\
15(26.8)\end{array}$ & 0.175 \\
\hline \multicolumn{7}{|l|}{$\mathrm{Bcl} 2$} \\
\hline $\begin{array}{l}\text { Negative } \\
\text { Positive }\end{array}$ & $\begin{array}{l}225(32.9) \\
459(67.1)\end{array}$ & $\begin{array}{l}29(45.3) \\
35(54.7)\end{array}$ & $0.045^{b}$ & $\begin{array}{l}221(32.2) \\
466(67.8)\end{array}$ & $\begin{array}{l}38(58.5) \\
27(41.5)\end{array}$ & $0.00002^{b}$ \\
\hline \multicolumn{7}{|l|}{ Bax } \\
\hline $\begin{array}{l}\text { Negative } \\
\text { Positive }\end{array}$ & $\begin{array}{l}309(69.9) \\
133(30.1)\end{array}$ & $\begin{array}{l}25 \text { (64.1) } \\
14(35.9)\end{array}$ & 0.45 & $\begin{array}{l}316(72.5) \\
120(27.5)\end{array}$ & $\begin{array}{l}20(46.5) \\
23(53.5)\end{array}$ & $0.0004^{b}$ \\
\hline
\end{tabular}

\section{HER2 Family}

EGFR

\begin{tabular}{|c|c|c|c|c|c|c|}
\hline $\begin{array}{l}\text { Negative } \\
\text { Overexpression }\end{array}$ & $\begin{array}{l}465(78.7) \\
126(21.3)\end{array}$ & $\begin{array}{l}39(70.9) \\
16(29.1)\end{array}$ & 0.183 & $\begin{array}{r}486(83.2) \\
98(16.8)\end{array}$ & $\begin{array}{l}39(69.6) \\
17(30.4)\end{array}$ & $0.011^{b}$ \\
\hline \multicolumn{7}{|l|}{ HER2 } \\
\hline $\begin{array}{l}\text { Negative } \\
\text { Overexpression }\end{array}$ & $\begin{array}{c}695(90.4) \\
74(9.6)\end{array}$ & $\begin{array}{l}56(81.2) \\
13(18.8)\end{array}$ & $0.016^{b}$ & $\begin{array}{r}676(89.3) \\
81(10.7)\end{array}$ & $\begin{array}{l}54(78.3) \\
15(21.7)\end{array}$ & $0.006^{b}$ \\
\hline \multicolumn{7}{|l|}{ HER3 } \\
\hline $\begin{array}{l}\text { Negative } \\
\text { Overexpression }\end{array}$ & $\begin{array}{l}292(48.4) \\
311(51.6)\end{array}$ & $\begin{array}{l}23(46.9) \\
26(53.1)\end{array}$ & 0.841 & $\begin{array}{l}321(52.7) \\
288(47.3)\end{array}$ & $\begin{array}{l}23(44.2) \\
29(55.8)\end{array}$ & 0.251 \\
\hline \multicolumn{7}{|l|}{ HER4 } \\
\hline $\begin{array}{l}\text { Negative } \\
\text { Overexpression }\end{array}$ & $\begin{array}{l}270(43.9) \\
345(56.1)\end{array}$ & $\begin{array}{l}25(50) \\
25(50)\end{array}$ & 0.404 & $\begin{array}{l}255(42.0) \\
352(58.0)\end{array}$ & $\begin{array}{l}23(41.8) \\
32(58.0)\end{array}$ & 1 \\
\hline \multicolumn{7}{|l|}{ Others } \\
\hline \multicolumn{7}{|c|}{ Triple-negative phenotype } \\
\hline $\begin{array}{l}\text { No } \\
\text { Yes }\end{array}$ & $\begin{array}{l}610(81.7) \\
137(18.3)\end{array}$ & $\begin{array}{l}49(71) \\
20(29)\end{array}$ & $0.027^{b}$ & $\begin{array}{l}629(83.6) \\
123(16.4)\end{array}$ & $\begin{array}{l}46(66.7) \\
23(33.3)\end{array}$ & $0.0004^{b}$ \\
\hline \multicolumn{7}{|c|}{ Basal-like phenotype } \\
\hline $\begin{array}{l}\text { No } \\
\text { Yes }\end{array}$ & $\begin{array}{l}599(83.7) \\
117(16.3)\end{array}$ & $\begin{array}{l}51(79.7) \\
13(20.3)\end{array}$ & 0.414 & $\begin{array}{l}626(85.6) \\
105(14.4)\end{array}$ & $\begin{array}{l}46(72.0) \\
18(28.0)\end{array}$ & $0.004^{b}$ \\
\hline \multicolumn{7}{|c|}{ 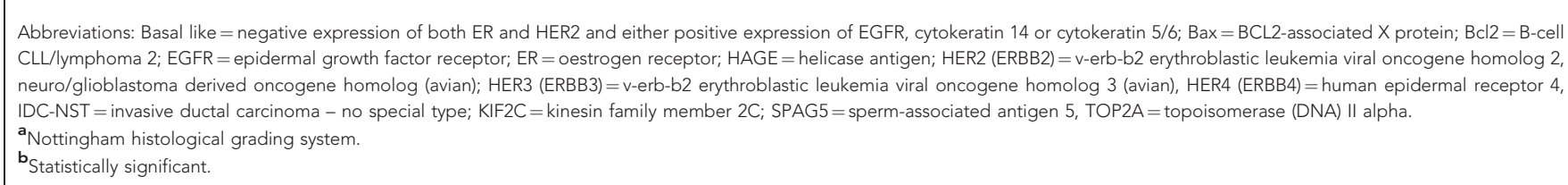 } \\
\hline
\end{tabular}

HAGE and other biomarkers of cell cycle and mitosis. HAGE + was significantly associated with high mitotic index (MI, $P=0.002)$, and high levels of Ki67 (Ki67 $+; P=0.01$ ), KIF2C $(\mathrm{KIF} 2 \mathrm{C}+; P=0.003)$ and SPAG5 (SPAG5 $+; P=0.01)$ expression. In the multivariate logistic analysis, high MI (OR (95\% CI); 1.5 (1.10-1.98), $P=0.018$ ) and low Bcl2 expression (OR (95\% CI); $0.33(0.20-0.56), P<0.0001)$ were independent predictors for HAGE + expression.

Survival analyses. In the whole cohort $(n=838)$, HAGE + expression was associated with adverse clinical outcomes at 10 years, with a significant increase in the risk of death $(P<0.0001)$ (Figure $1(\mathrm{~B}-1))$ and recurrence $(P=0.0002)$ compared with HAGE - tumours. Exploring the clinical outcome of patient subgroups with early-stage tumours (Figure 1(B-2)), ER + tumours (Figure 1(B-3)) or patients bearing ER - tumours (Figure 1(B-4)) confirmed that patients with HAGE + tumours exhibited a worse prognosis than those patients with tumours expressing low levels of HAGE.

Multivariate Cox regression analysis. HAGE, LN-stage, tumour size and $\mathrm{Bcl} 2$ were confirmed as powerful independent predictors for both BCSS and DFS in a multivariate Cox regression model, which included other validated traditional pathological prognostic factors and other co-founders and controlled for endocrine and chemotherapy $(P<0.01)$ (Table 2$)$.

\section{EP-BC patient group - test set $(n=838)$}

Clinico-pathological significance of HAGE expression. Eight percent (69 out of 838 ) of the tumours were HAGE + , and $92 \%$ (769 out of 838 ) tumours were HAGE - . HAGE + expression was 
Table 2. Multivariate Cox regression analysis in the discovery and test sets

BCSS at 10 years

DFS at 10 years

\begin{tabular}{|c|c|c|c|c|c|c|c|c|c|c|c|c|}
\hline \multirow[b]{2}{*}{ Variables } & \multicolumn{3}{|c|}{ Training set } & \multicolumn{3}{|c|}{ Test set } & \multicolumn{3}{|c|}{ Training set } & \multicolumn{3}{|c|}{ Test set } \\
\hline & HR & $95 \% \mathrm{Cl}$ & $P$ & HR & $95 \% \mathrm{Cl}$ & $P$ & HR & $95 \% \mathrm{Cl}$ & $P$ & HR & $95 \% \mathrm{Cl}$ & $P$ \\
\hline HAGE (high) & 2 & $1.3-3.1$ & $0.002^{*}$ & 1.9 & $1.3-2.9$ & $0.002^{*}$ & 1.7 & $1.2-2.5$ & $0.004^{*}$ & 2.5 & $1.8-3.5$ & $1 \times 10^{-7 x}$ \\
\hline \multicolumn{13}{|l|}{ Lymph node stage } \\
\hline $\begin{array}{l}\text { Negative } \\
\text { Positive (1-3 nodes) } \\
\text { Positive ( }>3 \text { nodes) }\end{array}$ & \begin{tabular}{|l|}
1 \\
1.3 \\
2.4
\end{tabular} & $\begin{array}{l}0.9-1.9 \\
1.5-3.9\end{array}$ & $0.002^{*}$ & $\begin{array}{l}1 \\
1.7 \\
4.6\end{array}$ & $\begin{array}{l}1.2-2.5 \\
2.8-7.2\end{array}$ & $6 \times 10^{-10 *}$ & $\begin{array}{l}1 \\
1.2 \\
2.9\end{array}$ & $\begin{array}{l}0.9-1.6 \\
1.9-4.4\end{array}$ & $1.4 \times 10^{-6 *}$ & $\begin{array}{l}1 \\
1.3 \\
2.7\end{array}$ & $\begin{array}{l}0.9-1.7 \\
1.8-4.1\end{array}$ & $7 \times 10^{-6 x}$ \\
\hline
\end{tabular}

Grade

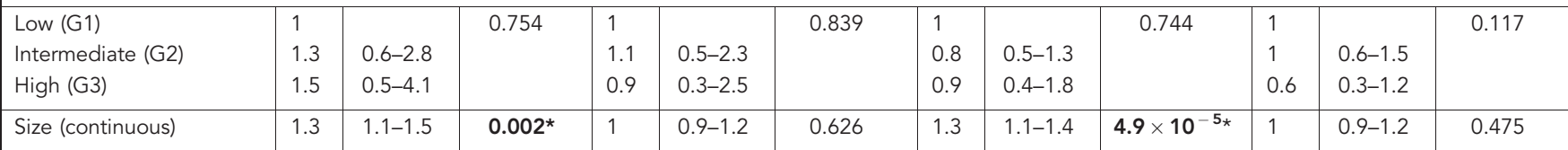

Mitotic index

\begin{tabular}{|c|c|c|c|c|c|c|c|c|c|c|c|c|}
\hline $\begin{array}{l}\text { M1 } \\
\text { M2 } \\
\text { M3 }\end{array}$ & $\begin{array}{l}1 \\
1.5 \\
1.8\end{array}$ & $\begin{array}{l}0.7-2.9 \\
0.8-4.3\end{array}$ & 0.4 & $\begin{array}{l}1 \\
1.2 \\
1.9\end{array}$ & $\begin{array}{l}0.7-2.4 \\
0.9-4.2\end{array}$ & 0.195 & $\begin{array}{l}1 \\
1.4 \\
1.5\end{array}$ & $\begin{array}{l}0.9-2.3 \\
0.8-3.0\end{array}$ & 0.362 & $\begin{array}{l}1 \\
1 \\
1.8\end{array}$ & $\begin{array}{l}0.6-1.6 \\
1.0-3.3\end{array}$ & $0.030 *$ \\
\hline Ki67 (high) & 1.6 & $1.0-2.5$ & 0.072 & 1.5 & $1.0-2.4$ & 0.082 & 1.1 & $0.8-1.6$ & 0.508 & 1.4 & $1.0-2.0$ & 0.079 \\
\hline $\mathrm{ER}(-)$ & 2.7 & $1.2-5.9$ & $0.015^{\star}$ & 1.1 & $0.6-2.4$ & 0.729 & 1.5 & $0.7-3.0$ & 0.288 & 1 & $0.5-1.9$ & 0.993 \\
\hline $\operatorname{HER} 2(+)$ & 1.8 & $1.0-3.2$ & $0.047 *$ & 1.4 & $0.8-2.5$ & 0.243 & 1.2 & $0.7-2.2$ & 0.473 & 1.1 & $0.6-1.8$ & 0.829 \\
\hline $\mathrm{Bcl} 2(-)$ & 2 & $1.4-2.9$ & $2 \times 10^{-4 \star}$ & 2.6 & $1.8-3.8$ & $2 \times 10^{-7 \star}$ & 1.5 & $1.1-2.0$ & 0.016 * & 2.3 & $1.7-3.1$ & $5 \times 10^{-8 \star}$ \\
\hline Triple negative (Yes) & 1.4 & $0.6-3.3$ & 0.492 & 1 & $0.4-2.4$ & 0.946 & 1 & $0.5-2.2$ & 0.984 & 0.7 & $0.4-1.5$ & 0.734 \\
\hline Endocrine therapy (Yes) & 0.8 & $0.6-1.2$ & 0.334 & 1 & $0.7-1.4$ & 0.969 & 0.9 & $0.7-1.2$ & 0.382 & 1 & $0.7-1.3$ & 0.915 \\
\hline Chemotherapy (Yes) & 0.7 & $0.5-1.1$ & 0.089 & 1 & $0.6-1.5$ & 0.879 & 0.8 & $0.6-1.1$ & 0.122 & 0.9 & $0.6-1.2$ & 0.479 \\
\hline
\end{tabular}

Abbreviations: $\mathrm{Bcl} 2=\mathrm{B}$-cell CLL/lymphoma 2; $\mathrm{BCSS}=$ breast cancer-specific survival; $\mathrm{Cl}=$ confidence interval; $\mathrm{DFS}=$ disease-free survival; $\mathrm{ER}=$ oestrogen receptor; $\mathrm{HAGE}=$ helicase antigen; HER2 = HER2 (ERBB2) = v-erb-b2 erythroblastic leukemia viral oncogene homolog 2, neuro/glioblastoma derived oncogene homolog (avian); HR = hazard ratio. M1 = low; mitoses $<10$, $\mathrm{M} 2=$ medium; mitoses $10-18, \mathrm{M} 3=$ high; mitoses $>18$. * Statistically significant $(P<0.05)$.

significantly associated with high histological grade $(P=0.0002)$, $\mathrm{ER}-/ \mathrm{PR}-(P<0.001), \mathrm{Bcl} 2-(P<0.0001), \mathrm{HER} 2+(P=0.006)$ and TNBC $(P=0.0004)$ (Table 1$)$. Statistical associations between HAGE + and high p16 $(P=0.002)$, high Bax $(P=0.0004)$, high EGFR $(P=0.01)$ and the presence of basal-like characteristics $(P=0.004)$ were observed in the test set but not in the training set, which has been confirmed when the two sets were pooled together. This observation could be due to the borderline imbalance between the test and training sets regarding the expression of EGFR $(P=050)$.

HAGE and other biomarkers of cell cycle and mitosis. HAGE + was significantly associated with high MI $(P<0.001)$, and high expression of Ki67 $(P=0.027), \operatorname{KIF} 2 \mathrm{C}(P=0.002)$ and SPAG5 $(P=0.004)$.

Survival analyses. HAGE + was found to be associated with adverse clinical outcomes in the whole cohort (Figure $2 \mathrm{~A} ; n=838$ ), as well as in subgroups that included the LN-negative patients (Figure 2B), ER + patients (Figure 2C) and ER - subpopulations (Figure 2D) $(<0.01)$. HAGE, LN stage, tumour size and $\mathrm{Bcl} 2$ were independent predictors for BCSS $(P<0.01)$ and DFS $(P<0.0001)$ in multivariate Cox regression analysis (Table 2 ).

HAGE is a prognostic biomarker in low-risk $(N P I \leqslant 3.4)$ $B C$ patients. The expression of HAGE + in low-risk $(\mathrm{NPI} \leqslant 3.4)$ $\mathrm{BC}$ patients who did not receive either endocrine or chemotherapy was associated with twice the risk of death $(\mathrm{HR}(95 \% \mathrm{CI})=1.8$
$(1.3-2.4), P=0.001)$ and relapse $(\mathrm{HR}(95 \% \mathrm{CI})=1.7(1.3-2.1)$, $P=0.000029)$.

HAGE is a prognostic biomarker in ER-positive BC patients. In high-risk/ER + BC, HAGE was associated with an adverse outcome at 10 years and an increased risk of death from BC (HR: 1.2; 95\% CI: $1.01-1.52 ; P=0.045$ ) and recurrence (HR: 1.4 ; 95\% CI: $1.2-1.7$; $P=0.0001)$. When high risk/ER $+\mathrm{BC}$ patients were stratified according to the status of hormone therapy, HAGE + retained its prognostic effect on DFS in patients who either received (HR: 1.4; 95\% CI: 1 . $1-1.8 ; P=0.009$ ) or did not receive hormonal therapy (HR: $1.5 ; 95 \%$ CI: $1.2-1.8 ; P=0.001$ ), whereas it lost its prognostic effect on BCSS in patients who did not receive hormonal therapy (HR: 0.96; 95\% CI: 0.6-1.4; $P=0.811$ ).

HAGE could be a predictor of hormone therapy resistance. ER + I high-risk BC patients who were HAGE - exhibited a prolonged BCSS and DFS when they were exposed to hormone therapy, compared with those who did not receive any hormone therapy $(P \mathrm{~s}<0.0001$; Figure $3 \mathrm{~A})$. No benefit was derived from prescribing hormone therapy for $\mathrm{ER}+/$ high-risk $\mathrm{BC}$ patients with $\mathrm{HAGE}+$ (Figure 3B).

PER-BC patient group. HAGE was highly expressed in $10 \%$ (27 out of 275) of the ER-negative cohort of patients with early stage disease (Figure $4 \mathrm{~A} ; n=275$ ).

Relationship between HAGE expression and clinical outcome in patients who received no anthracycline. Similar to our 

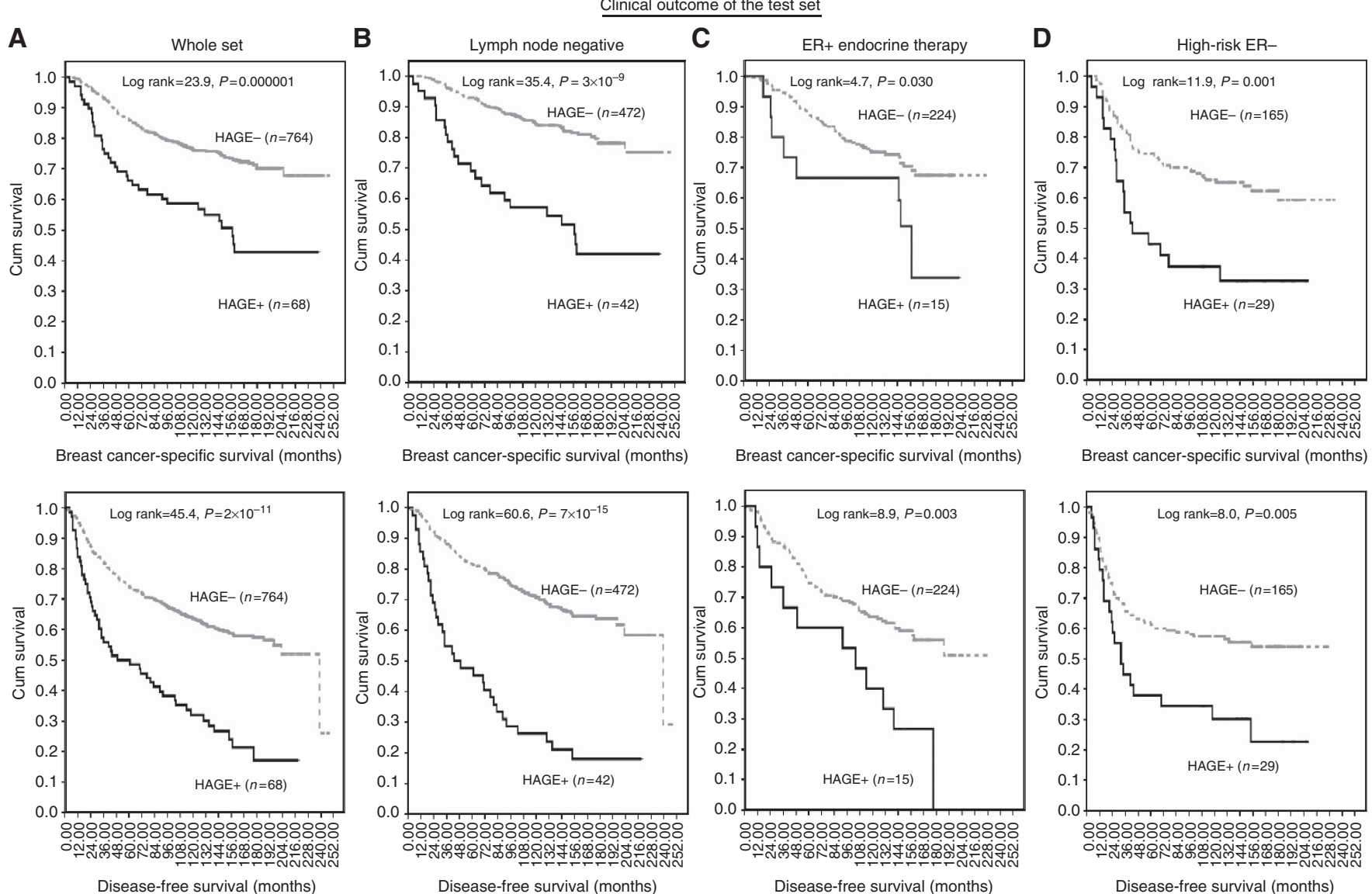

Figure 2. The clinical outcome of HAGE protein expression in the test set of primary early breast cancer (PE-BC). Kaplan-Meier curves showing the relationship between HAGE expression and breast cancer-specific survival and disease-free survival in the test set of primary early breast cancer (PE-BC). (A) Whole cohort; (B) LN - ; (C) ER + ; (D) high-risk ER - subpopulation. See text for details.

observation in the whole cohort (Figure 4A), HAGE + expression in patients with high-risk $\mathrm{ER}-\mathrm{BC}$ who did not receive chemotherapy or received less-effective CMF therapy $(n=134$; Figure 4B) was associated with adverse clinical outcomes at 5 years, with a significant increase in the risk of death $(P=0.0005)$ and recurrence $(P=0.0003)$ compared with HAGE - tumours.

Relationship between HAGE expression and clinical outcome in high risk/ER-negative $B C$ patients who received adjuvant-ACT. Patients whose tumour was found to be HAGE + and received adjuvant-ACT $(n=141)$ exhibited a similar risk of death, recurrence and distant metastases to those whose tumour was HAGE - (Figure 4C). This suggests a beneficial effect of ACT.

PLA-BC patients treated with neo-adjuvant ACT $(n=196)$. To validate the previous observation regarding the effect of HAGE expression and response to ACT, we investigated its expression in PLA-BC patients treated with neoadjuvant-ACT. All patients received neo-adjuvant-ACT, 63\% (123 out of 196) without and $37 \%$ (73 out of 196) with Taxane (Supplementary Table S3). Sixtythree percent of patients received six cycles of FEC (5-fluorouracil $500 \mathrm{mg}^{-2}$, epirubicin $75-100 \mathrm{mg}^{-2}$, cyclophosphamide $500 \mathrm{mg}^{-2}$, on day 1 of a 21 -day cycle).

Thirteen percent (26 out of 196) of PLA-BC patients achieved $\mathrm{pCR}$. The rates of pCR were higher for TNBC $(26 \% ; 14$ out of 55$)$ and the ER - /PR - /HER2 + subtypes (19\%; 3 out of 16) than for the $\mathrm{ER}+/ \mathrm{PR}+/ \mathrm{HER} 2-(8 \% ; 8$ out of 104$)$ and $\mathrm{ER}+/ \mathrm{PR}+1$ HER2 + (5\%; (1 out of 21$)$ subtypes; $P=0.009)$.
High prechemotherapy TIL scores were more frequent in the $\mathrm{ER}-/ \mathrm{PR}-/ \mathrm{HER} 2+(31 \% ; 5$ out of 16$)$ and TNBC $(27 \% ; 15$ out of 55$)$ subtypes than in the $\mathrm{ER}+/ \mathrm{PR}+/ \mathrm{HER} 2+(19 \% ; 4$ out of 21$)$ and $\mathrm{ER}+/ \mathrm{PR}+/ \mathrm{HER} 2-(11 \% ; 11$ out of 104$)$ subtypes $(P=0.029)$. Postchemotherapy surgical specimens of TNBC subtypes showed higher TIL (31\%; 17 out of 55) compared with $\mathrm{ER}-/ \mathrm{PR}-/ \mathrm{HER} 2+(12.5 \% ; 2$ out of 16$), \mathrm{ER}+/ \mathrm{PR}+1$ HER $2+(9.5 \% ; 2$ out of 21$)$ and ER + /PR + /HER2 - (11.5\%; 12 out of 104$)$ subtypes $(P=0.012)$. A high prechemotherapy TIL score was significantly associated with prechemotherapy HAGE + expression $(P=0.018)$.

Prechemotherapy TILs and HAGE expression are independent predictors of PCR rate for neoadjuvant-ACT. Among the 167 prechemotherapy core biopsies from patients with PLA-BC, $47 \%$ (78 out of 167) were HAGE +, and of those $24 \%$ (19 out of 78) achieved pCR. Tumours with prechemotherapy TIL + achieved higher pCR rates $(40 \%)$ compared with those with a TIL - status (7.5\%); $P<0.0001$. Sixty-five percent of prechemotherapy TIL + / HAGE + tumours achieved pCR, and the remaining 35\% were HAGE - in postchemotherapy specimens $(P<0.0001)$. In contrast, only 25,16 and $6 \%$ of the prechemotherapy TIL + /HAGE - , TIL $-/$ HAGE + and TIL $-/$ HAGE - tumours achieved pCR, respectively $(P<0.0001)$. Of note was the observation that $51 \%$ of prechemotherapy TIL - /HAGE + tumours were found to be HAGE - after receiving neoadjuvant chemotherapy. A statistically significant loss of HAGE expression following neoadjuvant chemotherapy was found among the 106 cases that were 
A
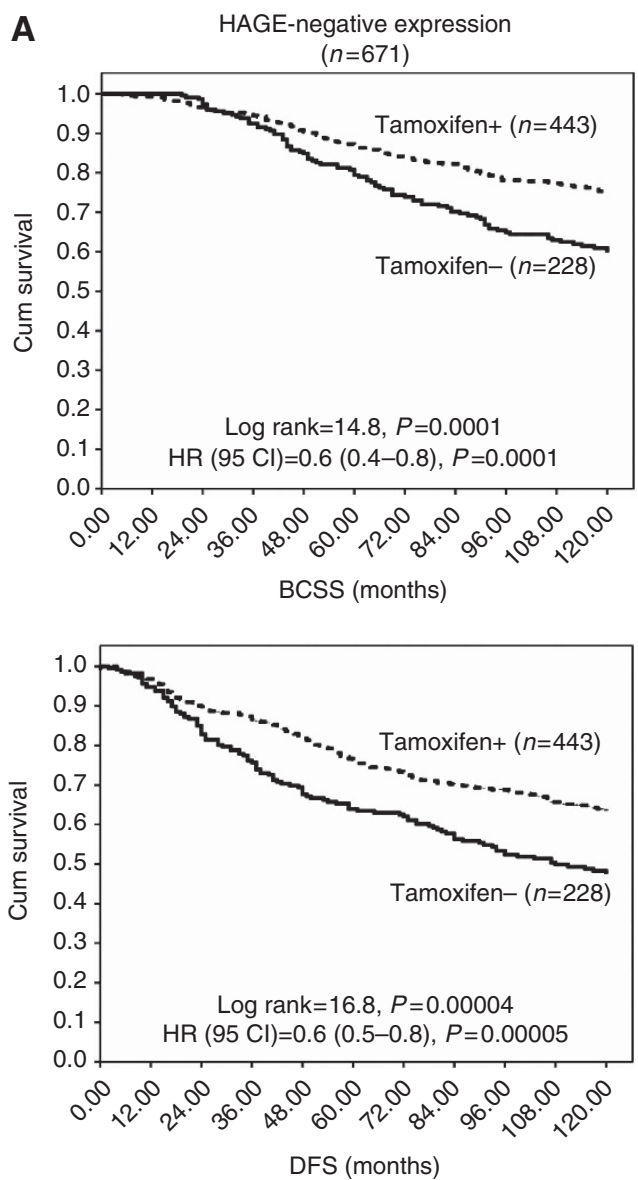

B
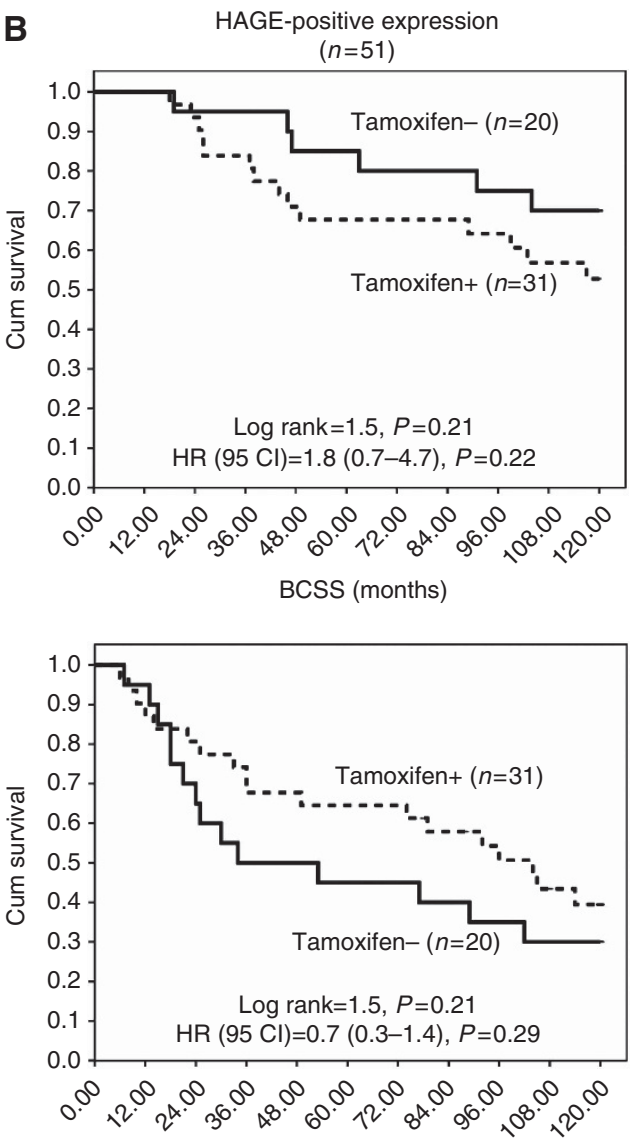

DFS (months)

Figure 3. The effect of Tamoxifen treatment on the clinical outcome of HAGE protein expression in high risk oestrogen receptor positive (ER +) primary early breast cancer (PE-BC). (A, B) Kaplan-Meier curves showing the effect of tamoxifen on breast cancer-specific survival (BCSS) and disease-free survival (DFS) in high-risk primary ER + breast cancer, stratified according to the HAGE status. (A) HAGE negative and (B) HAGE positive. See text for details.

paired prechemotherapy and postchemotherapy, $(P=0.000001$; Supplementary Table 5).

Multivariate logistic regression analysis showed that prechemotherapy TIL status (OR $(95 \% \quad \mathrm{CI})=5.9 \quad(1.8-20.8)$, $P=0.005)$ and HAGE expression $(\mathrm{OR}(95 \% \mathrm{CI})=4.6(1.3-16.4)$, $P=0.021)$ are independent predictors of $\mathrm{pCR}$ in patients with PLA-BC (Table 3).

Clinical outcome of HAGE expression and TILs status in PLA-BC. Patients with PLA-BC who received neoadjuvant-ACT and exhibited high levels of HAGE expression before chemotherapy exhibited a risk of progression which was similar to that in PLA-BC patients expressing low/no HAGE (log rank; 1.3, $P=0.250$, Figure 5(A-1)). However, ER - BC patients who received neoadjuvant ACT and exhibited $\mathrm{HAGE}+$ expression before chemotherapy were at a lower risk of progression than in those with HAGE - expression (log rank; 3.89, $P=.049$, Figure 5(A-2)).

In contrast, patients with $\mathrm{HAGE}+$ residual disease after neoadjuvant-ACT exhibited a worse progression-free survival (log rank; 12.8, $P=0.0003$ ) than those in whom HAGE expression was absent (Figure $5(B-1)$ ). This was especially the case for patients with ER + tumours (log rank; 7.6, $P=0.006$ ); Figure 5(B-2).

High prechemotherapy TIL status was significantly associated with increased BCSS (HR, 0.07; 95\% CI, 0.01 to $0.51 ; P=0.009$ ) and DFS (HR, 0.05 ; $95 \% \mathrm{CI}, 0.01$ to $0.36 ; P=0.003$ ), whereas high postchemotherapy TIL status was associated with increases in DFS (HR, 0.43 ; 95\% CI, 0.19 to $0.94 ; P=0.033$ ) and a trend towards an increased BCSS ( $\mathrm{HR}, 0.41 ; 95 \% \mathrm{CI}, 0.16$ to $1.05 ; P=0.062$ ).
The 5-year DFS rates for the high prechemotherapy TILs + / HAGE + (90\%) and TILs + /HAGE - (86\%) subgroups were higher than for those with TILs - /HAGE + (53\%) and TILs - / HAGE $-(46 \%) ; P=0.001$.

\section{DISCUSSION}

To the best of our knowledge, this is the first study to report on the prognostic and predictive values of HAGE expression in primary BC. The clinically significant findings of this study are that: (a) HAGE + expression is significantly associated with aggressive clinico-pathological and high proliferation parameters and acts as a novel independent factor for poor prognosis; (b) HAGE + expression identifies a subgroup of PER-BC and PER + BC patients who could benefit from ACT and are resistant to tamoxifen; respectively; and (c) TILs and HAGE + expression in prechemotherapy biopsies are independent predictors of PCR of PLA-BC. Because of the high level of HAGE protein expression in tumours compared with normal tissues, its association with lymphocyte infiltration and the previously reported immunogenic potential of HAGE (Mathieu et al, 2007), this antigen could be proposed as a novel immunotherapeutic target for this sub-group of BC patients with HAGE + (Mathieu et al, 2010). Work is currently ongoing to confirm that HAGE-specific T cells can indeed kill HAGE-positive BC cells.

In the current study, high levels of HAGE expression was observed in $8-10 \%$ of patients with $\mathrm{BC}$ and was associated with 
A
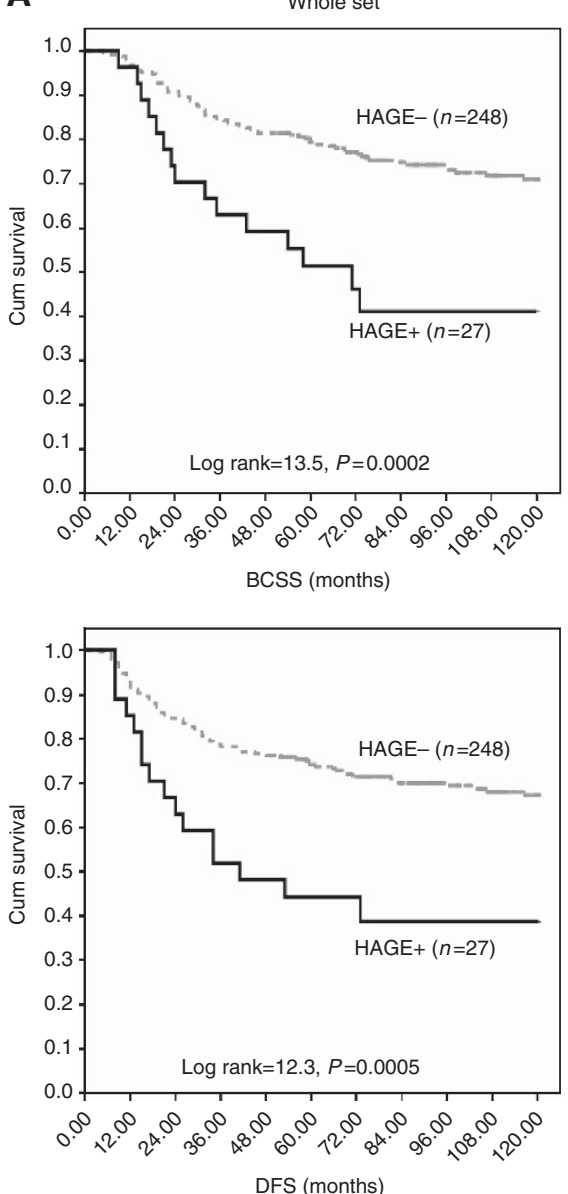

B
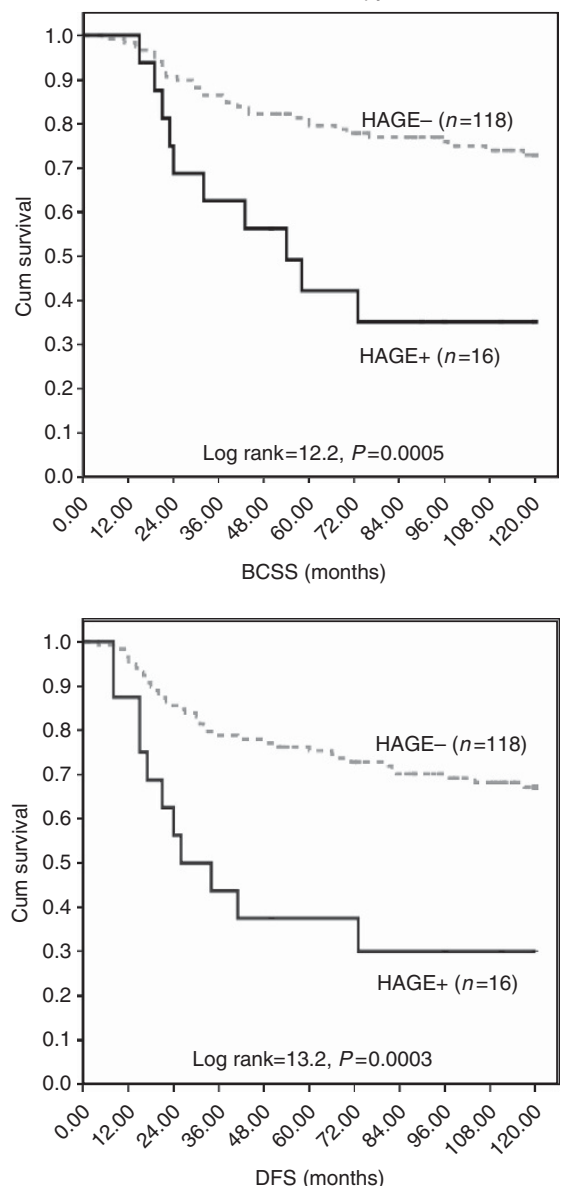

C
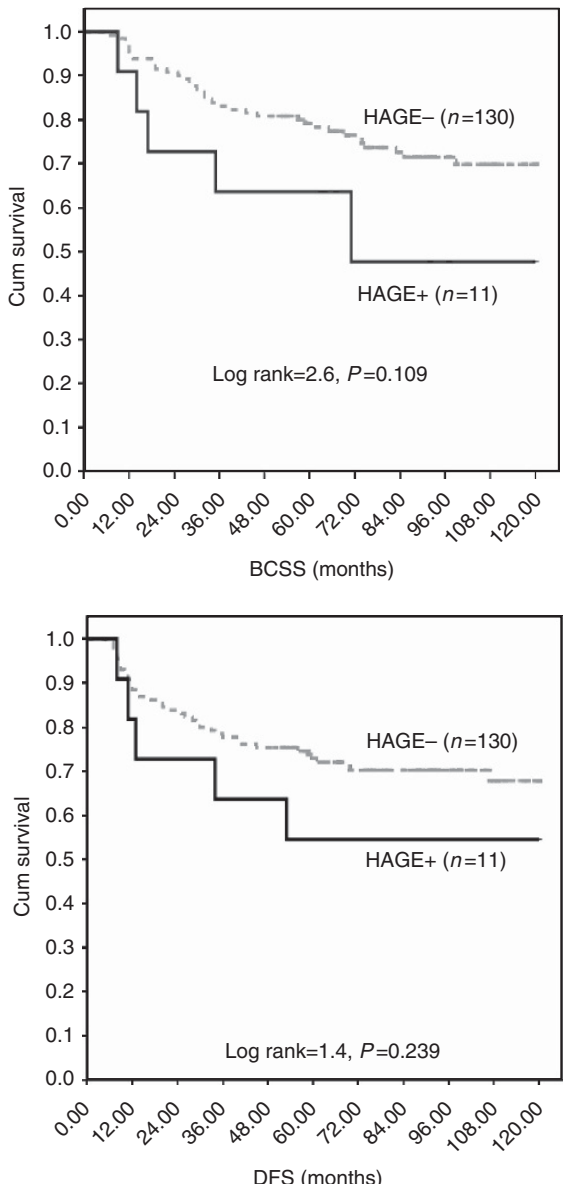

Figure 4. The clinical outcome of HAGE protein expression in high risk oestrogen receptor negative (ER-) primary early breast cancer (PE-BC). (A-C) Kaplan-Meier curves showing the relationship between HAGE expression and breast cancer-specific survival (BCSS) and disease-free survival (DFS) in primary oestrogen receptor-negative breast cancer (PER-BC), stratified according to the received adjuvant chemotherapy protocols.

(A) Whole cohort, (B) no chemotherapy/or less effective CMF and (C) anthracycline combination therapy (ACT). See text for details.

Table 3. Multivariate regression analysis

\begin{tabular}{|l|c|c|c|}
\hline Factors & OR & $95 \%$ Cl & $P$ \\
\hline Tumour-infiltrating lymphocyte(TIL+) & 5.92 & $1.69-20.78$ & $0.005^{\mathrm{a}}$ \\
\hline Prechemotherapy HAGE (+) & 4.55 & $1.26-16.44$ & $0.021^{\mathrm{a}}$ \\
\hline Top2A (+) & 4.53 & $1.10-18.88$ & $0.038^{\mathrm{a}}$ \\
\hline Triple negative (+) & 3.1 & $0.84-11.46$ & 0.091 \\
\hline Clinical TNM stage (II vs III) & 0.97 & $0.22-4.25$ & 0.964 \\
\hline Tumour grade (G1/2 vs G3)** & 0.4 & $0.13-1.25$ & 0.114 \\
\hline $\begin{array}{l}\text { Maximum diameter of prechemotherapy } \\
\text { tumour size (continuous) }\end{array}$ & 1.01 & $0.99-1.04$ & 0.422 \\
\hline Patient age (continuous) & 1.03 & $0.96-1.09$ & 0.426 \\
\hline $\begin{array}{l}\text { Abbreviations: Cl=confidence interval; HAGE = helicase antigen; OR=odds ratio; TNM }= \\
\text { Tumour, Node, Metastases; TOP2A =topoisomerase (DNA) II alpha. TIL, HAGE and TOP2A } \\
\text { are independent predictors for pathological complete response. }{ }^{* * N o t t i n g h a m ~ h i s t o l o g i c a l ~} \\
\text { grading system. } \\
\text { a Statistically significant. }\end{array}$
\end{tabular}

increased risk of recurrence and metastases. Moreover, an increase in the percentage of HAGE-positive tumours was found between early and locally advanced disease. These findings, along with our previously published data (Linley et al, 2012), support the concept that HAGE is implicated in the progression of the disease by driving cell proliferation and potentially the metastasis of $\mathrm{BC}$ cells. HAGE has been shown to be overexpressed at both the mRNA and protein level at varying frequencies in different human tumours (Martelange et al, 2000; Adams et al, 2002; Mathieu et al, 2010) compared with matched normal tissue, with the exception of testes. Furthermore, in agreement with previous studies on other CTA (Kurashige et al, 2001; Otte et al, 2001; Yakirevich et al, 2003; Lurquin et al, 2005; Roman-Gomez et al, 2007; Chen et al, 2011b), we have found that HAGE + expression was associated with TNT, less-differentiated, higher-grade tumours and poorer clinical outcome. However, the exact molecular mechanisms underlying the increase of HAGE expression in human cancer remain unclear.

There is substantial evidence that epigenetic events represent one mechanism that regulates the expression of HAGE, and global DNA methylation seems to have a major role (Roman-Gomez et al, 2007). It has previously been shown that hypomethylation of the HAGE gene promoter correlates with increased HAGE expression and is strongly associated with advanced disease and poor prognosis of leukaemia. This supports the potential role of HAGE in increased cellular proliferation and its potential as a marker of disease progression (Roman-Gomez et al, 2007). Interestingly, the global hypomethylation of DNA and histones has been shown to progressively increase in parallel with advanced grade and disease progression in human cancers (Kim et al, 1994; Qu et al, 1999; Soares et al, 1999; Piyathilake et al, 2001). 
A

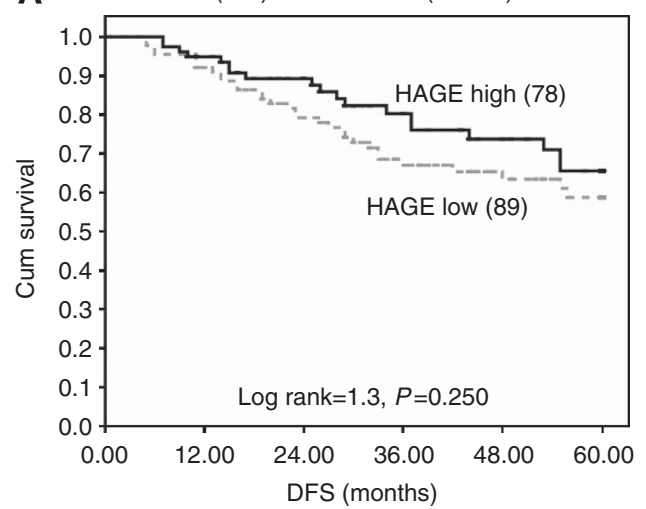

B

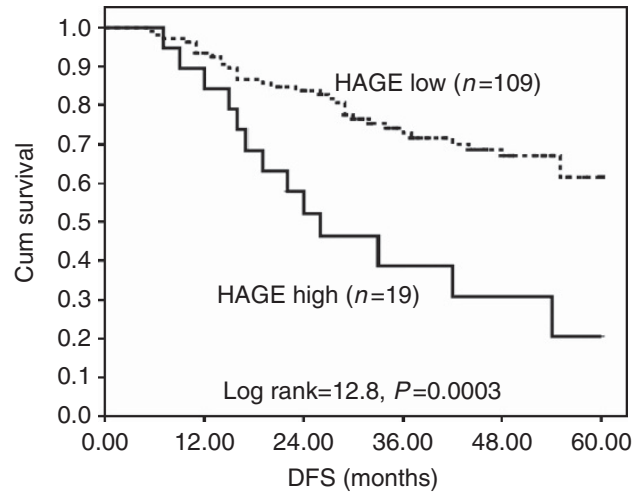

(A-2) ER- cases cohort $(n=76)$

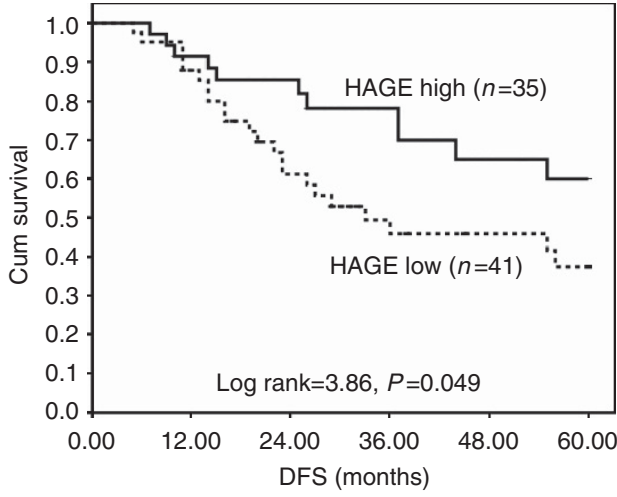

(B-2) ER+ cases cohort $(n=71)$

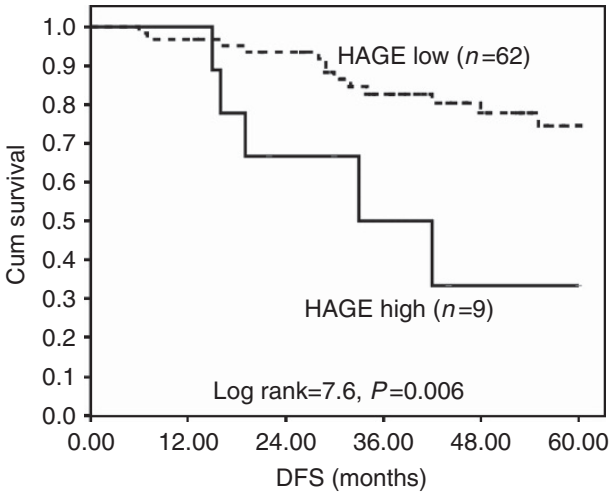

Figure 5. The clinical outcome of HAGE protein expression in primary locally advanced breast cancer (PLA-BC) before and after receiving neoadjuvant chemotherapy. (A) Kaplan-Meier curves illustrating the relationship between prechemotherapy expression of HAGE and disease-free survival (DFS) in the entire PLA-BC cohort (A-1) and in the ER - subgroup (A-2). (B) Kaplan-Meier curves illustrating the relationship between HAGE expression and DFS in patients who exhibited evidence of pathological residual disease after receiving neoadjuvant-ACT in the entire PLA-BC cohort (B-1) and in the ER+ subgroup (B-2).

The precise function of HAGE in human cancer remains unknown. We have recently confirmed that HAGE is involved in promoting proliferation via the Ras signalling pathway. This was illustrated by observations that transfection of cells with a HAGE cDNA construct increased cell proliferation of cells and that the silencing of HAGE reduced proliferation (Linley et al, 2012). Moreover, preliminary results using shRNA to permanently knock down HAGE expression also suggest the involvement of HAGE in tumour motility and metastasis (data not shown). Roman-Gomez et al (2007) have speculated that HAGE expression might confer a selective advantage over HAGE - cells in blast crisis in leukaemia. Although this would explain the selection of HAGE + cells during the progression of leukaemia, the possible role of HAGE in the transformed status of $\mathrm{BC}$ cells needs to be clarified in further functional studies.

In the present study, survival analysis indicates that HAGE is an independent prognostic factor for EP-BC patients. This finding is consistent with the finding in leukaemia, in which $\mathrm{HAGE}+$ transcripts have been associated with poor prognosis (Roman-Gomez et al, 2007; Chen et al, 2011a). Similarly, for a given cancer type, higher expression of some other CTAs, such as testes-specific protease 50 (TSP 50) for colorectal (Zheng et al, 2011), MAGE-A3 for pancreatic cancer (Kim et al, 2006), MAGE-C2 for hepatocellular carcinoma (Riener et al, 2009) and NY-ESO-1 for malignant melanoma (Velazquez et al, 2007), were often correlated with poorer outcomes. Recently, Germain et al (2011) found that expression of DEAD-box 1 mRNA is an independent prognostic marker for the early recurrence of $\mathrm{BC}$.
The protein encoded by the HAGE gene is a member of the family of 'DEAD-box proteins', which function as important transcriptional regulators and are involved in many aspects of RNA metabolism, spermatogenesis, embryogenesis and cell growth (Iggo et al, 1990; Yang et al, 2005; Roman-Gomez et al, 2007). The DEAD-box RNA helicases p68 (DDX5) and p72 (DDX17) have been shown to act as transcriptional co-activators for a diverse range of transcription factors, including oestrogen receptor $\alpha$ $(\mathrm{ER} \alpha)$, and a role for them in miRNA maturation, ribosome biogenesis, mRNA splicing and insulator function have also been described (Shin et al, 2007; Wortham et al, 2009). IHC staining of $\mathrm{ER} \alpha$-positive primary $\mathrm{BC}$ for these DEAD-box proteins indicated that p72 expression was associated with prolonged DFS and OS as well as being inversely associated with HER2 expression. Our studies have found HAGE + expression to be associated with ERnegative expression and other aggressive phenotypes, such as TNBC, HER2 overexpression, proliferation and high grade. Furthermore, HAGE + expression was associated with DFS and BCSS and tamoxifen residence in ER + tumours. However, further functional studies to confirm these preliminary data are warranted.

HAGE expression could prove to be a useful biomarker in the stratification of patients with BC for chemotherapy, as our data show that $\mathrm{HAGE}+$ patients may benefit from ACT. It can be postulated that cells expressing HAGE proliferate faster and are therefore more responsive to ACT. A recent study (Zheng et al, 2011) has shown that cell proliferation, colony formation, migration and cell apoptosis were reduced/inhibited and cell sensitivity to doxorubicin enhanced, when the TSP50 gene expression was silenced. Moreover, the fact that patients with HAGE + residual 
disease (ACT-resistant cells) exhibited a poorer outcome suggests that alternative therapy such as immunotherapy may be warranted for this group. Zitvogel et al (2008) have highlighted that anthracyclines, unlike many other cytotoxic agents, can elicit immunogenic cell death and potentiate the potency of vaccines.

The DNA methyltransferase inhibitor 5-aza- $2^{\prime}$-deoxycytidine (decitabine) has been shown to be beneficial for patients with chronic myeloid leukaemia (Issa et al, 2004). Drugs that are capable of inducing HAGE expression could therefore increase the eligibility of patients for CTA-targeted immunotherapy and improve the management of patients with recurrent disease.

\section{CONFLICT OF INTEREST}

TMAA-F, SEBM, RCR and SYTC are named inventors on a PCT patent application that is jointly held by the NHS Trust and Nottingham Trent University (Patent publication number WO2013144616 published on 03.10.2013). The other authors declare no conflict of interest.

\section{STATEMENT OF TRANSLATIONAL RELEVANCE}

Tissues from three cohorts of patients: early primary disease (EP-BC; $n=1676$ ), primary ER-negative disease (PER-BC; $n=275$ ) and primary locally advanced disease (PLA-BC; $n=196)$ were evaluated for the expression of the cancer testes antigen HAGE (CT13, DDX43) and the presence of tumourinfiltrating lymphocytes (TILs) before and after chemotherapy. HAGE expression and the presence of TILs were found to be significantly associated with aggressive clinico-pathological features and to act as independent factors for poor prognosis. Moreover, high HAGE expression (HAGE + ) and the presence of TILs identified a subgroup of breast cancer patients (ER - ) who would benefit from neo-adjuvant anthracycline combination therapies (Neo-adjuvant-ACT). Furthermore, given the high level of HAGE protein expression in tumours compared with normal tissues, the strong association of HAGE expression with the presence of TILs and our previously reported data on the immunogenic potential of HAGE (Mathieu et al, 2007), this antigen might be a novel immunotherapeutic target for patients with HAGE + breast cancer and for patients with other HAGE + cancers.

\section{AUTHOR CONTRIBUTIONS}

SYTC, TMAA-F, SEBM and RCR designed the study. SYTC, TMAA-F, SEBM and RCR, GRB, PMM, AGP and IOE were involved in drafting the manuscript and took part in critically reviewing it for publication. SYTC, TMAA-F, SEBM, GRB and IOE analysed and interpreted the data. PMM and CJ performed the immunohistochemical staining of experimental slides. IOE and TMAA-F undertook the pathological assessment of experimental slides. PMM conducted collection and management of patient data. Figures, tables and referencing were generated by TMAA-F.

\section{REFERENCES}

Abdel-Fatah TM, Powe DG, Agboola J, Adamowicz-Brice M, Blamey RW, Lopez-Garcia MA, Green AR, Reis-Filho JS, Ellis IO (2010a) The biological, clinical and prognostic implications of p53 transcriptional pathways in breast cancers. J Pathol 220(4): 419-434.

Abdel-Fatah TM, Powe DG, Ball G, Lopez-Garcia MA, Habashy HO, Green AR, Reis-Filho JS, Ellis IO (2010b) Proposal for a modified grading system based on mitotic index and Bcl2 provides objective determination of clinical outcome for patients with breast cancer. J Pathol 222(4): 388-399.

Adams SP, Sahota SS, Mijovic A, Czepulkowski B, Padua RA, Mufti GJ, Guinn BA (2002) Frequent expression of HAGE in presentation chronic myeloid leukaemias. Leukemia 16(11): 2238-2242.

Chen Q, Lin J, Qian J, Yao DM, Qian W, Li Y, Chai HY, Yang J, Wang CZ, Zhang M, Xiao GF (2011a) Gene expression of helicase antigen in patients with acute and chronic myeloid leukemia. Zhongguo Shi Yan Xue Ye Xue Za Zhi 19(5): 1171-1175.

Chen YT, Ross DS, Chiu R, Zhou XK, Chen YY, Lee P, Hoda SA, Simpson AJ, Old LJ, Caballero O, Neville AM (2011b) Multiple cancer/testis antigens are preferentially expressed in hormone-receptor negative and high-grade breast cancers. PLoS One 6(3): e17876.

Denkert C, Loibl S, Noske A, Roller M, Müller BM, Komor M, Budczies J, Darb-Esfahani S, Kronenwett R, Hanusch C, von Törne C, Weichert W, Engels K, Solbach C, Schrader I, Dietel M, von Minckwitz G (2010) Tumor-associated lymphocytes as an independent predictor of response to neoadjuvant chemotherapy in breast cancer. J Clin Oncol 28(1): 105-113.

Dotan E, Goldstein LJ (2010) Optimizing chemotherapy regimens for patients with early-stage breast cancer. Clin Breast Cancer 10(Suppl 1): E8-15.

Early Breast Cancer Trialists' Collaborative GroupClarke M, Coates AS, Darby SC, Davies C, Gelber RD, Godwin J, Goldhirsch A, Gray R, Peto R, Pritchard KI, Wood WC (2008) Adjuvant chemotherapy in oestrogenreceptor-poor breast cancer: patient-level meta-analysis of randomised trials. Lancet 371(9606): 29-40.

Elston CW, Ellis IO (1991) Pathological prognostic factors in breast cancer. I. The value of histological grade in breast cancer: experience from a large study with long-term follow-up. Histopathology 19(5): 403-410.

Germain DR, Graham K, Glubrecht DD, Hugh JC, Mackey JR, Godbout R (2011) DEAD box 1: a novel and independent prognostic marker for early recurrence in breast cancer. Breast Cancer Res Treat 127(1): 53-63.

Gluck S, Mamounas T (2010) Improving outcomes in early-stage breast cancer. Oncology 24(11 Suppl 4): 1-15.

Hammond ME, Hayes DF, Wolff AC, Mangu PB, Temin S (2010) American Society of Clinical Oncology/College of American Pathologists guideline recommendations for immunohistochemical testing of estrogen and progesterone receptors in breast cancer. J Oncol Pract 6(4): 195-197.

Harbeck N, Salem M, Nitz U, Gluz O, Liedtke C (2010) Personalized treatment of early-stage breast cancer: present concepts and future directions. Cancer Treat Rev 36(8): 584-594.

Harris L, Fritsche H, Mennel R, Norton L, Ravdin P, Taube S, Somerfield MR, Hayes DF, Bast Jr RC. American Society of Clinical O (2007) American Society of Clinical Oncology 2007 update of recommendations for the use of tumor markers in breast cancer. J Clin Oncol 25(33): 5287-5312.

Iggo R, Picksley S, Southgate J, McPheat J, Lane DP (1990) Identification of a putative RNA helicase in E.coli. Nucleic Acids Res 18(18): 5413-5417.

Issa JP, Garcia-Manero G, Giles FJ, Mannari R, Thomas D, Faderl S, Bayar E, Lyons J, Rosenfeld CS, Cortes J, Kantarjian HM (2004) Phase 1 study of low-dose prolonged exposure schedules of the hypomethylating agent 5-aza-2'-deoxycytidine (decitabine) in hematopoietic malignancies. Blood 103(5): 1635-1640.

Kim J, Reber HA, Hines OJ, Kazanjian KK, Tran A, Ye X, Amersi FF, Martinez SR, Dry SM, Bilchik AJ, Hoon DS (2006) The clinical significance of MAGEA3 expression in pancreatic cancer. Int J C 118(9): 2269-2275.

Kim YI, Giuliano A, Hatch KD, Schneider A, Nour MA, Dallal GE, Selhub J, Mason JB (1994) Global DNA hypomethylation increases progressively in cervical dysplasia and carcinoma. Cancer 74(3): 893-899.

Kurashige T, Noguchi Y, Saika T, Ono T, Nagata Y, Jungbluth A, Ritter G, Chen YT, Stockert E, Tsushima T, Kumon H, Old LJ, Nakayama E (2001) Ny-ESO-1 expression and immunogenicity associated with transitional cell carcinoma: correlation with tumor grade. Cancer Res 61(12): 4671-4674.

Linley AJ, Mathieu MG, Miles AK, Rees RC, McArdle SE, Regad T (2012) The helicase HAGE expressed by malignant melanoma-initiating cells is required for tumor cell proliferation in vivo. J Biol Chem 287(17): 13633-13643.

Lurquin C, Lethe B, De Plaen E, Corbiere V, Theate I, van Baren N, Coulie PG, Boon T (2005) Contrasting frequencies of antitumor and 
anti-vaccine $\mathrm{T}$ cells in metastases of a melanoma patient vaccinated with a MAGE tumor antigen. J Exp Med 201(2): 249-257.

Martelange V, De Smet C, De Plaen E, Lurquin C, Boon T (2000) Identification on a human sarcoma of two new genes with tumor-specific expression. Cancer Res 60(14): 3848-3855.

Mathieu MG, Knights AJ, Pawelec G, Riley CL, Wernet D, Lemonnier FA, Straten PT, Mueller L, Rees RC, McArdle SE (2007) HAGE, a cancer/testis antigen with potential for melanoma immunotherapy: identification of several MHC class I/II HAGE-derived immunogenic peptides. Cancer Immunol Immunother 56(12): 1885-1895.

Mathieu MG, Linley AJ, Reeder SP, Badoual C, Tartour E, Rees RC, McArdle SE (2010) HAGE, a cancer/testis antigen expressed at the protein level in a variety of cancers. Cancer Immun 10: 2 .

McShane LM, Altman DG, Sauerbrei W, Taube SE, Gion M, Clark GM. Statistics Subcommittee of the NCIEWGoCD (2005) Reporting recommendations for tumor marker prognostic studies (REMARK). J Natl Cancer Inst 97(16): 1180-1184.

Otte M, Zafrakas M, Riethdorf L, Pichlmeier U, Loning T, Janicke F, Pantel K (2001) MAGE-A gene expression pattern in primary breast cancer. Cancer Res 61(18): 6682-6687.

Pinder SE, Ellis IO, Galea M, O’Rouke S, Blamey RW, Elston CW (1994) Pathological prognostic factors in breast cancer. III. Vascular invasion: relationship with recurrence and survival in a large study with long-term follow-up. Histopathology 24(1): 41-47.

Piyathilake CJ, Frost AR, Bell WC, Oelschlager D, Weiss H, Johanning GL, Niveleau A, Heimburger DC, Grizzle WE (2001) Altered global methylation of DNA: an epigenetic difference in susceptibility for lung cancer is associated with its progression. Hum Pathol 32(8): $856-862$.

Qu G, Dubeau L, Narayan A, Yu MC, Ehrlich M (1999) Satellite DNA hypomethylation vs. overall genomic hypomethylation in ovarian epithelial tumors of different malignant potential. Mutat Res 423(1-2): 91-101.

Riener MO, Wild PJ, Soll C, Knuth A, Jin B, Jungbluth A, Hellerbrand C, Clavien PA, Moch H, Jochum W (2009) Frequent expression of the novel cancer testis antigen MAGE-C2/CT-10 in hepatocellular carcinoma. Int J Cancer 124(2): 352-357.

Roman-Gomez J, Jimenez-Velasco A, Agirre X, Castillejo JA, Navarro G, San Jose-Eneriz E, Garate L, Cordeu L, Cervantes F, Prosper F, Heiniger A, Torres A (2007) Epigenetic regulation of human cancer/testis antigen gene, HAGE, in chronic myeloid leukemia. Haematologica 92(2): $153-162$.

Shin S, Rossow KL, Grande JP, Janknecht R (2007) Involvement of RNA helicases p68 and p72 in colon cancer. Cancer Res 67(16): 7572-7578.
Soares J, Pinto AE, Cunha CV, Andre S, Barao I, Sousa JM, Cravo M (1999) Global DNA hypomethylation in breast carcinoma: correlation with prognostic factors and tumor progression. Cancer 85(1): $112-118$.

Velazquez EF, Jungbluth AA, Yancovitz M, Gnjatic S, Adams S, O’Neill D, Zavilevich K, Albukh T, Christos P, Mazumdar M, Pavlick A, Polsky D, Shapiro R, Berman R, Spira J, Busam K, Osman I, Bhardwaj N (2007) Expression of the cancer/testis antigen NY-ESO-1 in primary and metastatic malignant melanoma (MM) - correlation with prognostic factors. Cancer Immun 7: 11.

Wolff AC, Hammond ME, Schwartz JN, Hagerty KL, Allred DC, Cote RJ, Dowsett M, Fitzgibbons PL, Hanna WM, Langer A, McShane LM, Paik S, Pegram MD, Perez EA, Press MF, Rhodes A, Sturgeon C, Taube SE, Tubbs R, Vance GH, van de Vijver M, Wheeler TM, Hayes DF. American Society of Clinical O, College of American P (2007) American Society of Clinical Oncology/College of American Pathologists guideline recommendations for human epidermal growth factor receptor 2 testing in breast cancer. J Clin Oncol 25(1): 118-145.

Wortham NC, Ahamed E, Nicol SM, Thomas RS, Periyasamy M, Jiang J, Ochocka AM, Shousha S, Huson L, Bray SE, Coombes RC, Ali S, Fuller-Pace FV (2009) The DEAD-box protein p72 regulates ERalpha-/ oestrogen-dependent transcription and cell growth, and is associated with improved survival in ERalpha-positive breast cancer. Oncogene 28(46): 4053-4064.

Yakirevich E, Sabo E, Lavie O, Mazareb S, Spagnoli GC, Resnick MB (2003) Expression of the MAGE-A4 and NY-ESO-1 cancer-testis antigens in serous ovarian neoplasms. Clin Cancer Res 9(17): 6453-6460.

Yang L, Lin C, Liu ZR (2005) Phosphorylations of DEAD box p68 RNA helicase are associated with cancer development and cell proliferation. Mol Cancer Res 3(6): 355-363.

Zheng L, Xie G, Duan G, Yan X, Li Q (2011) High expression of testes-specific protease 50 is associated with poor prognosis in colorectal carcinoma. PLoS One 6(7): e22203.

Zitvogel L, Apetoh L, Ghiringhelli F, Kroemer G (2008) Immunological aspects of cancer chemotherapy. Nat Rev Immunol 8(1): 59-73.

This work is published under the standard license to publish agreement. After 12 months the work will become freely available and the license terms will switch to a Creative Commons AttributionNonCommercial-Share Alike 3.0 Unported License.

Supplementary Information accompanies this paper on British Journal of Cancer website (http://www.nature.com/bjc) 